\title{
Dust enshrouded star-forming activity in Arp 299*
}

\author{
P. Gallais ${ }^{1}$, V. Charmandaris ${ }^{2,3}$, E. Le Floc' ${ }^{1}$, I. F. Mirabel ${ }^{1,4}$, M. Sauvage ${ }^{1}$, L. Vigroux ${ }^{1}$, and O. Laurent ${ }^{1}$ \\ ${ }^{1}$ Service d'Astrophysique, CEA-Saclay, 91191 Gif sur Yvette Cedex, France \\ 2 Cornell University, Astronomy Department, Ithaca, NY 14853, USA \\ ${ }^{3}$ Chercheur Associé, Observatoire de Paris, 61 Av. de l'Observatoire, 75014 Paris, France \\ ${ }^{4}$ Instituto de Astronomía y Física del Espacio/CONICET, cc 67, suc 28, 1428 Buenos Aires, Argentina
}

Received 5 May 2003 / Accepted 18 September 2003

\begin{abstract}
We present mid-infrared spectro-imaging $(5-16 \mu \mathrm{m})$ observations of the infrared luminous interacting system Arp 299 (= Mrk 171 = IC 694+NGC 3690) obtained with the ISOCAM instrument aboard ISO. Our observations show that nearly $40 \%$ of the total emission at 7 and $15 \mu \mathrm{m}$ is diffuse, originating from the interacting disks of the galaxies. Moreover, they indicate the presence of large amounts of hot dust in the main infrared sources of the system and large extinctions toward the nuclei. While the observed spectra have an overall similar shape, mainly composed of Unidentified Infrared Bands (UIB) in the short wavelength domain, a strong continuum at $\sim 13 \mu \mathrm{m}$ and a deep silicate absorption band at $10 \mu \mathrm{m}$, their differences reveal the varying physical conditions of each component. For each source, the spectral energy distribution (SED) can be reproduced by a linear combination of a UIB "canonical" spectral template and a hot dust continuum due to a 230-300 K black body, after independently applying an extinction correction to both of them. We find that the UIB extinction does not vary much throughout the system $\left(A_{\mathrm{V}} \lesssim 5 \mathrm{mag}\right)$ suggesting that most UIBs originate from less enshrouded regions. IC 694 appears to dominate the infrared emission of the system and our observations support the interpretation of a deeply embedded nuclear starburst located behind an absorption of about 40 magnitudes. The central region of NGC 3690 displays a hard radiation field characterized by a $[\mathrm{NeIII}] /[\mathrm{NeII}]$ ratio $\geq 1.8$. It also hosts a strong continuum from 5 to $16 \mu \mathrm{m}$ which can be explained as thermal emission from a deeply embedded $\left(A_{\mathrm{V}} \sim 60 \mathrm{mag}\right)$ compact source, consistent with the mid-infrared signature of an active galactic nucleus $(\mathrm{AGN})$, and in agreement with recent $\mathrm{X}$-ray findings.
\end{abstract}

Key words. stars: formation - galaxies: individual: Arp 299 - galaxies: individual: Mrk 171 - galaxies: interactions galaxies: starburst - infrared: ISM

\section{Introduction}

At a distance of $41 \mathrm{Mpc}\left(v_{\text {hel }}=3080 \mathrm{~km} \mathrm{~s}^{-1}, H_{0}=\right.$ $75 \mathrm{~km} \mathrm{~s}^{-1} \mathrm{Mpc}^{-1}$ ), Arp 299 (Mrk $171=$ IC $694+$ NGC 3690) is one of the nearest interacting galaxies. Because of its proximity, its spatial extent $(8 \mathrm{kpc})$, and its high infrared (IR) luminosity $^{1}\left(L_{\mathrm{IR}}=5.16 \times 10^{11} L_{\odot}\right)$, it is one of the prime candidates for exploring the effects of triggered star formation activity as the tidal forces during an interaction lead to instabilities in the galactic disks and rapidly funnel large quantities of gas into the dynamical centers of the galaxies (Mihos \& Hernquist 1996; Sanders \& Mirabel 1996). Similar to the prototypical interacting pair of galaxies NGC 4038/39, this system is dynamically

Send offprint requests to: $\mathrm{P}$. Gallais,

e-mail: pascal.gallais@cea.fr

* Based on observations with ISO, an ESA project with instruments funded by ESA Member States (especially the PI countries: France, Germany, The Netherlands and the UK) with the participation of ISAS and NASA.

${ }^{1}$ We use the standard definition of $L_{\mathrm{IR}}(8-1000 \mu \mathrm{m})=5.62 \times$ $10^{5} D_{\mathrm{Mpc}}^{2}\left(13.48 f_{12}+5.16 f_{25}+2.58 f_{60}+f_{100}\right) L_{\odot}$ (see Sanders \& Mirabel 1996). young, as shown by the prominent $180 \mathrm{kpc}\left(13^{\prime}\right)$ long tidal tail (Hibbard \& Yun 1999).

Arp 299 has been studied extensively at all wavelengths over the past thirty years (see the original work by Weedman 1972). Most notably though, the mid-infrared and radio work of Gehrz et al. (1983), the observations in the optical by Augarde \& Lequeux (1985), in the near-infrared by Gallais (1991) and Wynn-Williams et al. (1991), along with the information on its molecular gas kinematics by Aalto et al. (1997) and Casoli et al. (1999) have provided ample evidence for the extreme characteristics of this system. More recently, the high spatial resolution images obtained with the Hubble Space Telescope (HST) in the near-IR (Alonso-Herrero et al. 2000), as well as in the mid-IR using Keck (Soifer et al. 2001) have revealed numerous point-like sources that were undetected in the optical, and firmed up our understanding of the complex starforming history of the galaxy (see Alonso-Herrero et al. 2000, and references therein). Following the notation suggested by Gehrz et al. (1983), the system is usually described by the galaxy IC 694 (source A) to the east, the nuclear region of NGC 3690 (source B) to the south-west and two regions of 


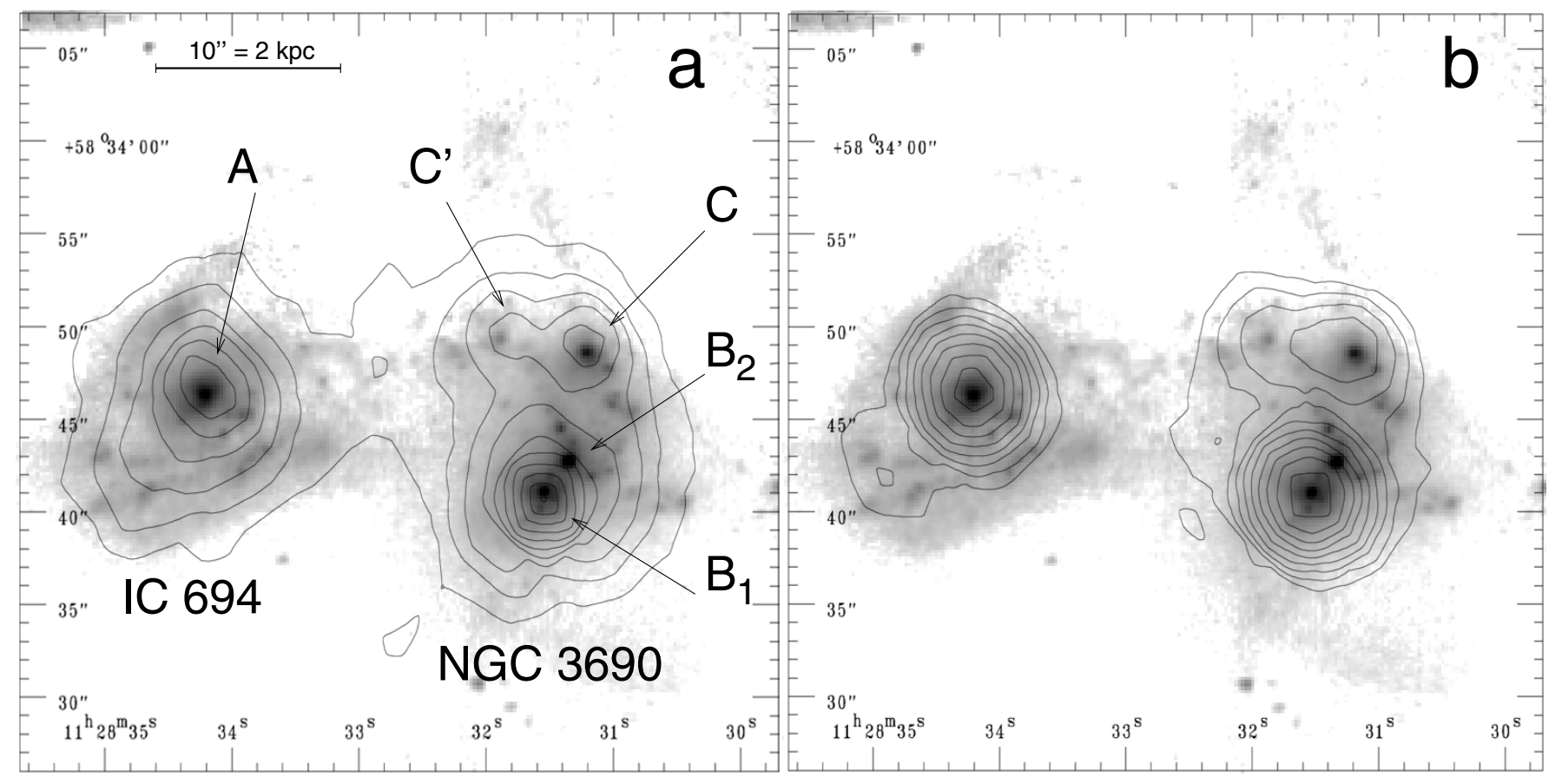

Fig. 1. a) The HST/NICMOS $2.2 \mu \mathrm{m}$ image of Arp 299 from Alonso-Herrero et al. (2000) with the overlay of the ISOCAM $7 \mu \mathrm{m}$ emission. The different components of the system are indicated. The 9 contour levels are set with logarithmic spacing between 1 and $33 \mathrm{mJy}$ arcsec ${ }^{-2}$. The image scale is displayed with a bar of $2 \mathrm{kpc}\left(10^{\prime \prime}\right)$ at the top. b) Same as in a) but using the ISOCAM $15 \mu \mathrm{m}$ image as an overlay, having set the contour limits to 6 and $60 \mathrm{mJy} \operatorname{arcsec}^{-2}$. Adapted from Charmandaris et al. (2002).

emission (sources $\mathrm{C}$ and $\mathrm{C}^{\prime}$ ) to the north-west. Source B in NGC 3690 has been further resolved into several components, among which B1 and B2 clearly dominate the global emission in the near- and mid-IR (see Fig. 1 for details). Interestingly it was also recently shown by Charmandaris et al. (2002) that despite its inconspicuous appearance in the optical, IC 694 contributes nearly half of the infrared $(8-1000 \mu \mathrm{m})$ luminosity of the system.

One of the recurrent questions though in all studies of Arp 299 is whether this galaxy is a pure starburst as was proposed by Weedman (1972) or whether some luminosity could be contributed by Active Galactic Nucleus (AGN). The fact that it is one of the most X-ray luminous galaxies $\left(L_{0.2-4.0 \mathrm{keV}} \sim\right.$ $10^{42} \mathrm{rg} \mathrm{s}^{-1}$, see Fabbiano et al. 1992; Zezas et al. 1998) along with the flatness of its radio continuum emission had given some indications that an enshrouded AGN could be lurking in one of the nuclei. Only recently though the hard X-ray (10-40 keV) Beppo-SAX observations of Della Ceca et al. (2002) have provided unambiguous evidence that a deeply buried $\left(N_{\mathrm{H}}=2.5 \times 10^{24} \mathrm{~cm}^{-2}\right)$ AGN is located in one of the interacting components of the system. Furthermore, the same authors also demonstrated that the intrinsic AGN luminosity in both the UV and X-rays is almost an order of magnitude less than the infrared emission of the system calculated by Charmandaris et al. (2002), indicating that the bulk of the infrared emission of the galaxy is indeed due to massive star formation as proposed by Laurent et al. (2000). The poor spatial resolution of the Beppo-SAX can not pinpoint the exact location of the AGN. However, preliminary analysis of the Chandra data (Zezas et al. 2003), which provide sub-arcsecond resolution, suggests that the AGN might be located within source B1 of NGC 3690 .

Motivated by this recent activity, we decided to analyze in detail our 5-16 $\mu$ m ISOCAM (Cesarsky et al. 1996) spectrophotometric data on this system. Our observations provide good spatial resolution with unprecedent sensitivity and enable us to examine in detail the dust properties of the obscured nuclear regions of Arp 299. The observations and data reduction methods are described in Sect. 2, a brief description of the global mid-IR morphology of the system is presented in Sect. 3 and the analysis of the mid-IR spectra is shown in Sect. 4. In Sect. 5, we discuss the implications of our findings on the physical characteristics of the various infrared sources and our conclusions are summarized in Sect. 6.

\section{Observations and data reduction}

Arp 299 was part of the ISO guaranteed time program CAMACTIV (PI. I.F. Mirabel) which had, as a prime goal, the study of the mid-IR properties of more than 20 nearby active/interacting galaxies (Laurent et al. 2000). It has been observed with ISOCAM with the Continuously Variable Filter (CVF), resulting in a full coverage of the spectral range from 5 to $16 \mu \mathrm{m}$ with a spectral resolution $R \sim 40$. The pixel size was $1.5^{\prime \prime}$ giving a total field of view of $48^{\prime \prime} \times 48^{\prime \prime}$. The system was then fully mapped with an effective spatial resolution ranging from 3 to $4.5^{\prime \prime}$. The data reduction and analysis were performed using the 
CAM Interactive Software (CIA) ${ }^{2}$. Dark subtraction was done using a model of the secular evolution of ISOCAM's dark current. Cosmic rays were removed using a multi-resolution median filtering method while the memory effects of the detector were corrected using the so-called IAS transient correction algorithm which is based on an inversion method (Abergel et al. 1996). The flat field correction was performed using the library of calibration data. Finally, individual exposures were combined using shift techniques in order to correct the effect of jittering due to the satellite motions (amplitude $\sim 1^{\prime \prime}$ ). These methods and their consequences are discussed in detail in Starck et al. (1999). We estimate that the uncertainty of our mid-IR photometric measurements is $\sim 20 \%$.

Experience with past ISOCAM observations has indicated that the overall pointing of ISO in the CVF mode is reliable. However, given the disturbed morphology of the system, we verified the relative astrometry by comparing and identifying the main features of our mid-IR images with those detected at other wavelengths after taking into account the shape of their spectral energy distribution (SED) as well as possible effects of absorption. For this purpose, we made an extensive use of the HST WFPC2 and NICMOS data of Alonso-Herrero et al. (2000), as well as the Keck $12 \mu$ m images obtained by Soifer et al. (2001). Although these mid-IR data are not as deep as our ISOCAM observations, their much higher spatial resolution clearly facilitated our efforts. Since one can not blindly rely on the astrometry information provided by the ISO telemetry, we based our identification on the nucleus of IC 694 (source A), which is sufficiently isolated and its position is well defined in both the HST near-IR images as well as in the Keck $12 \mu \mathrm{m}$ maps. Finally the stability of the roll angle of the telescope during the observations and the apriori knowledge of the separation of the brighter mid-IR point sources enabled us to securely identify the position of the remaining components.

\section{The Mid-IR morphology}

As mentioned earlier, the comparison between our data and the high resolution Keck images of Soifer et al. (2001) allowed us to clearly locate the four components $\mathrm{A}, \mathrm{B}, \mathrm{C}$ and $\mathrm{C}^{\prime}$ as the dominant sources of emission in our ISOCAM images. These identifications further revealed that the centroid of our source B actually corresponds the position of B1. Even though the angular separation between B1 and B2 is only 2", well below our spatial resolution, the fact that $\mathrm{B} 2$ is only marginally detected in the maps of Soifer et al. (2001) leads us to believe that its contribution to the mid-IR emission of B is minimal longward of $7 \mu \mathrm{m}$. Thus the conclusions we will draw for source B actually reflect the characteristics of B1.

In an attempt to increase our sensitivity to faint emission features, we used the CVF dataset to construct broad band images at the two most commonly used ISOCAM filters centered at 7 and $15 \mu \mathrm{m}$. The resulting $5-8.5 \mu \mathrm{m}$ and $12-18 \mu \mathrm{m}$ images,

${ }^{2}$ The ISOCAM data presented in this paper were analyzed using "CIA", a joint development by the ESA Astrophysics Division and the ISOCAM Consortium led by the ISOCAM PI, C. Cesarsky, Direction des Sciences de la Matière, CEA, France.
Table 1. Broad-band mid-IR photometry of Arp 299.

\begin{tabular}{lccc}
\hline \hline Source & LW $^{\dagger}(\mathrm{mJy})$ & LW $^{\dagger}(\mathrm{mJy})$ & LW3/LW2 \\
\hline $\mathrm{A}$ & 325 & 1860 & 5.72 \\
$\mathrm{~B}$ & 505 & 1951 & 3.86 \\
$\mathrm{C}$ & 126 & 461 & 3.66 \\
$\mathrm{C}^{\prime}$ & 76 & 232 & 3.05 \\
Total & 1846 & 7037 & 3.81 \\
\hline
\end{tabular}

$\dagger$ Using a beam of $4.5^{\prime \prime}$ in diameter (aperture corrections were applied to account for the overall extension of the PSF).

which correspond to the LW2 and LW3 filters, reach a $1 \sigma$-rms noise of 0.27 and $0.44 \mathrm{mJy} \mathrm{arcsec}^{-2}$ respectively. They are presented in Fig. 1, adapted from Charmandaris et al. (2002), with their contours overlaid on the NICMOS HST data obtained by Alonso-Herrero et al. (2000). The LW2 image is dominated by the contribution of the Unidentified Infrared Band (UIB) features and as a result traces mostly the contribution of photodissociation and quiescent star-forming regions. The LW3 image, on the other hand, mainly samples the thermal signature of hot dust from massive star-forming regions as well as emission from high excitation forbidden lines such as [NeII] $12.8 \mu \mathrm{m}$ and [NeIII]15.6 $\mu \mathrm{m}$. A more detailed description on how the underlying mid-IR SED affects the LW2 and LW3 colors in active galaxies is presented in Laurent et al. (2000). The mid-IR fluxes of the various sources are reported in Table 1.

In addition to the four main components (A, B, C and $\mathrm{C}^{\prime}$ ) detected in our mid-IR images, we note that there is considerable diffuse emission, in particular at $7 \mu \mathrm{m}$, in the region between IC 694 and NGC 3690 as well as around the nuclei of the galaxies. Our equivalent broad band images reveal that this diffuse component, which was undetected in the shallower ground based images, accounts for $44 \%$ and $36 \%$ of the total 7 and $15 \mu \mathrm{m}$ fluxes originating from Arp 299. This diffuse emission is shown in Fig. 2 which displays normalized profiles at $7.1 \mu \mathrm{m}$ (continuum) and $7.7 \mu \mathrm{m}$ (UIB band) of sources A and B1 compared to the PSF profiles. Together with the profiles, images of the diffuse emission at these wavelengths are presented, obtained by subtraction of a synthetic image made of PSFs positioned at the locations of sources A, B1, C and $\mathrm{C}^{\prime}$ to the images of the system in the $7.7 \mu \mathrm{m}$ UIB and in its continuum. If we derive an equivalent IRAS $12 \mu \mathrm{m}$ flux assuming a power-law SED in the 5-18 $\mu \mathrm{m}$ range, we find that $60 \%$ of the overall IRAS $12 \mu \mathrm{m}$ flux is accounted for by the four sources, with A and B1 being the dominant ones (22 and $28 \%$ of the total flux respectively).

All main components in the system are very bright in both filters and have extremely red colors. The 15 to $7 \mu \mathrm{m}$ flux ratios LW3/LW2 range from 3 to 5.7 and they are quite high compared with what is typically found in normal galaxies where they vary from 0.7 to 1.2 (Roussel et al. 2001). Even in the case of the Antennae galaxies, Knot A, the brightest $15 \mu \mathrm{m}$ region, exhibits a LW3/LW2 ratio of 2.6 (Mirabel et al. 1998), while the same indicator in the massive star-forming complex within the outer ring of the Cartwheel galaxy is 5.2 (Charmandaris et al. 1999). In the absence of an AGN-type activity, such high ratios in Arp 299 indicate a star-forming mechanism of a remarkably high efficiency (Gallais et al. 1999; Laurent et al. 2000), 

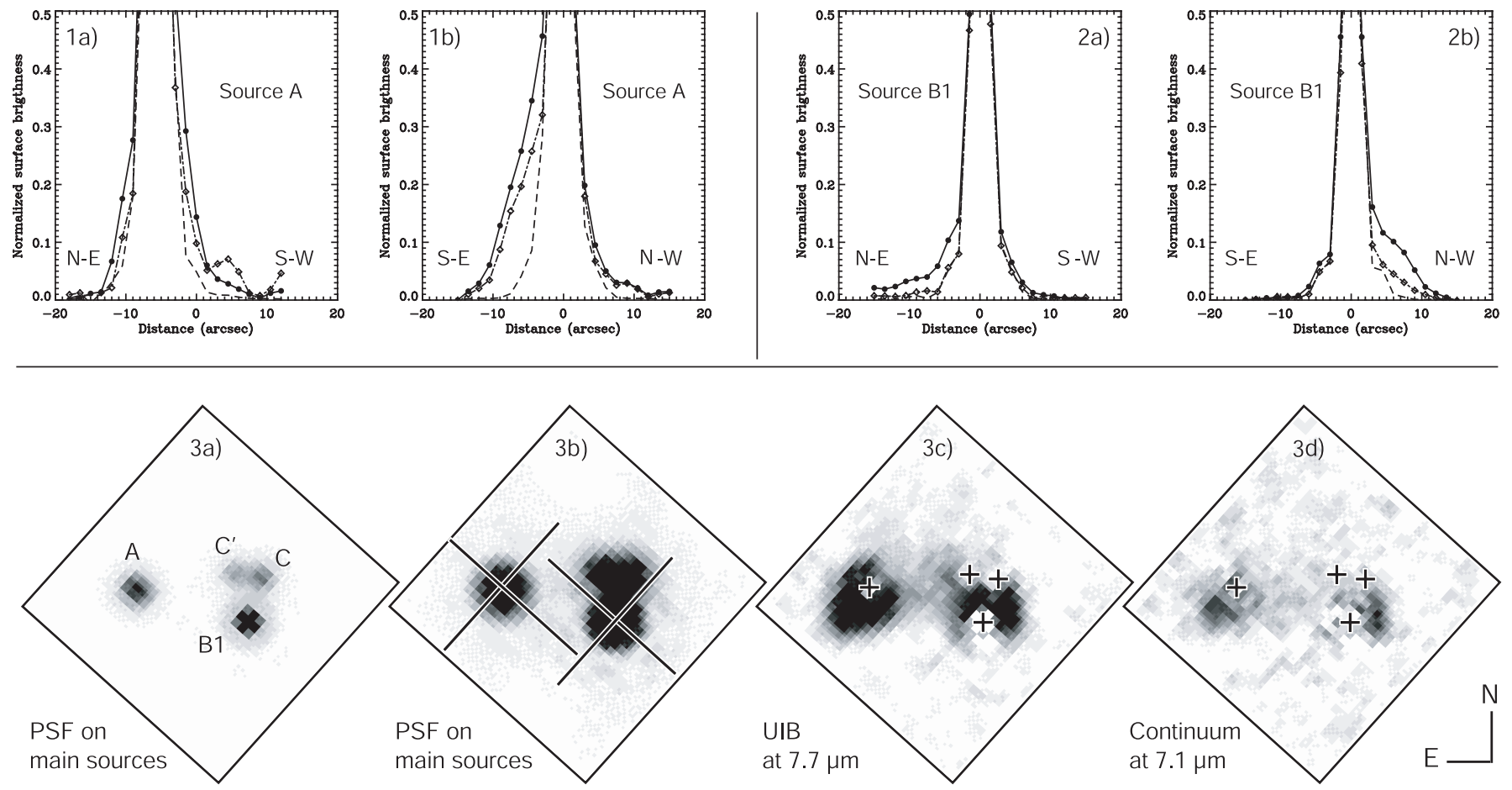

Fig. 2. Normalized surface brigthness profiles of sources A (panels 1a), 1b)) and B1 (panels 2a), 2b)) in the continuum at $7.1 \mu \mathrm{m}($ dot-dashed line with diamonds) and in the UIB band at $7.7 \mu \mathrm{m}$ (filled dots on continuous line) overplotted to the normalized profile of the PSF (dashed line) along the 2 detector axis (considered pixels are tagged by a line in image 3b)). Image 3a) and 3b) represent synthetic image built with adjusted PSFs positioned at the locations of $\mathrm{A}, \mathrm{B} 1, \mathrm{C}$ and $\mathrm{C}^{\prime}$ (3a): full dynamical range; 3b): 5\% of the maximum). Images 3c) and 3d) display the extended emission respectively in the $7.7 \mu \mathrm{m}$ UIB and in the $7.1 \mu \mathrm{m}$ continuum, i.e. the residual emission after subtraction of the synthetic image of PSFs to the image of the system in both bands (same dynamical range than the one applied for image $\mathbf{3 b}$ )).

a result which has also been suggested by Lai et al. (1999) and Rouan et al. (1999) based on their near-IR adaptive optics observations. Using a gray body model to fit the mid- and far-IR SED of the system, Charmandaris et al. (2002) have estimated that the infrared luminosities of the three main components $(\mathrm{A}$, $\mathrm{B} 1+\mathrm{B} 2$ and $\mathrm{C}+\mathrm{C}^{\prime}$ ) range from 0.44 to $1.8 \times 10^{11} L_{\odot}$. This implies that each of these sources is several times more luminous than most starburst galaxies observed in the local Universe such as the prototypical M 82.

Examining the individual sources in more detail, it is striking that, within the limits of our resolution, component $\mathrm{A}$ is dominated in the mid-IR by a strong nuclear source with a faint extension towards the southeast. This nuclear source is completely absent from the optical HST images and only becomes apparent in the original near-IR maps of Wynn-Williams et al. (1991). In the near-IR, this is interpreted as the emission from the old stellar population in the bulge of IC 694. However, the mid-IR spectrum (see Fig.4) differs clearly from the one of a population of old stars, such as in elliptical galaxy, in which there is no emission or absorption band (Athey et al. 2002). It is rather similar to the one we encounter in star forming regions (Laurent et al. 2000). In the mid-IR, the dominant process is star formation which is not evidenced at shorter wavelengths (visible and near-IR) and it appears mainly concentrated in the nuclear region. At longer wavelengths, the emission of $\mathrm{A}$ dominates the global infrared emission of the system, making A the most luminous component of the system at $38 \mu \mathrm{m}$ (Charmandaris et al. 2002). This implies that even though star formation does happen over an extended area of IC 694 - as evidenced by the dust lanes and star forming tidal streamers visible in the HST optical imagery of Malkan et al. (1998) - and does contribute in the mid-IR by creating an extended emission seen in Fig. 1, the bulk of the infrared flux originates from its nucleus.

The mid-IR structure of NGC 3690 is more complex. As discussed in detail by Alonso-Herrero et al. (2000), a large number of point-like sources can be seen in the near-IR, with B1 and B2 largely dominating the overall emission of the galaxy at these wavelengths. The spectral properties of these two main components though present a remarkable contrast. As the wavelength increases, B1 becomes progressively brighter while the contribution of B2 - clearly the most luminous source of NGC 3690 in the visible and in the $J$ and $H$ bands (Alonso-Herrero et al. 2000) - gently declines longward of $1.6 \mu \mathrm{m}$. Furthermore, the latter is barely detectable in the high resolution mid-IR images of Soifer et al. (2001), while B1 clearly unveils a very luminous core at $12 \mu \mathrm{m}$. The red colors of $\mathrm{B} 1$ and its differential extinction of $A_{\mathrm{V}}=15$ mag relative to B2 (Alonso-Herrero et al. 2000) are indicative of its much more obscured nature and reveal the presence of large concentrations of dust and gas in this area. As a side note, it is worth mentioning that B1 is completely invisible in the HST/FOC $220 \mathrm{~nm} \mathrm{UV}$ map of the galaxy (see Fig. 3), and it is very faint at $300 \mathrm{~nm}$ (see Windhorst et al. 2002, Fig. 3.34a,b), an additional evidence of its high dust content. 


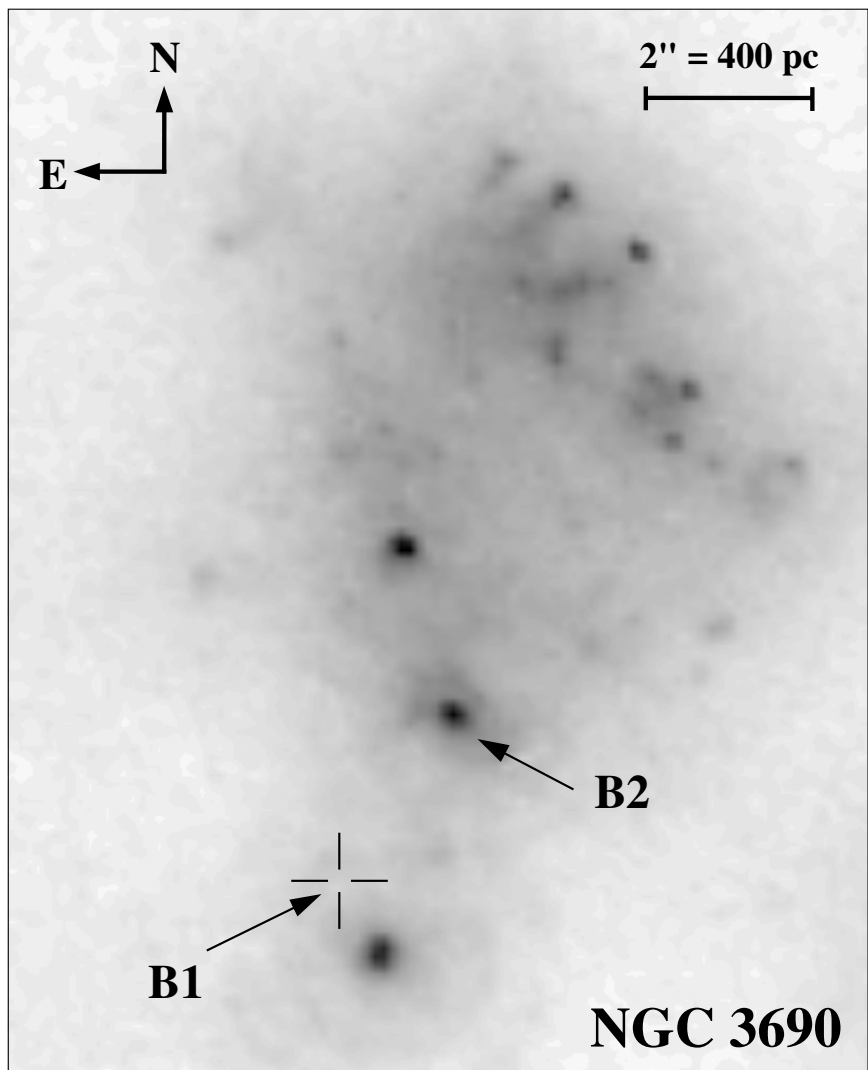

Fig. 3. UV image ( $220 \mathrm{~nm}$ ) of NGC 3690 taken with the FOC on-board the HST (see also Meurer et al. (1995)) with positions of B1 and B2 marked. One can see that no emission at this wavelength is associated with B1 confirming its highly obscured nature.

Sources $C$ and $C^{\prime}$ are clearly resolved at $7 \mu \mathrm{m}$ but the emission from $\mathrm{C}^{\prime}$ diminishes relatively to $\mathrm{C}$ at longer wavelengths (see Fig. 1). It has been proposed that source $C$ could be the nucleus of a third galaxy in the interacting system, but this hypothesis is questioned by the location of this source on the $[J-H] /[H-K]$ color-color diagram (Gallais 1991), far from the locus of the other nuclei. Recent CO observations (Aalto et al. 1997; Casoli et al. 1999) also suggest that $C$ and $C^{\prime}$ are dynamically linked and can be considered as star-forming complexes in the overlap region of NGC 3690 and IC 694.

\section{Mid-IR spectral properties and absorption}

In Fig. 4, we present the 5-16 $\mu \mathrm{m}$ spectra of the four main sources A, B1, C and C' of Arp 299. They have been obtained with a $4.5^{\prime \prime} \times 4.5^{\prime \prime}$ aperture centered on the brightest spot of each component. A wavelength-dependent scaling factor has also been applied to correct for the extended flux of the PSF lying outside of the aperture used. We observe that the overall shape of each spectrum is similar, with the UIB features dominating the emission in the short wavelength range and with a warm continuum becoming visible longward of $10 \mu \mathrm{m}$. Our measurements are also consistent with the $8-13 \mu \mathrm{m}$ spectroscopic observations already obtained by Dudley (1999).

A more thorough analysis though, reveals distinct underlying physical properties of each region. While the mid-IR SED of source $\mathrm{C}$ is typical of what is generally observed in starburst galaxies (e.g., Laurent et al. 2000), the spectra of sources A and $C^{\prime}$ display very strong absorption in the $9.7 \mu \mathrm{m}$ silicate band and a significant contribution of the thermal continuum at $\lambda>12 \mu \mathrm{m}$. Sources $\mathrm{C}$ and $\mathrm{C}^{\prime}$ also display a strong [NeIII] emission line, which as we discuss in Sect. 5, is not as apparent in the other components. Finally, we note that the spectrum of source B1 is rather intriguing since it is dominated by an important hot dust continuum throughout the whole CVF spectral range, with only a minor contribution of the UIB features.

To provide a more quantitative insight into the properties of these sources, their 5-16 $\mu \mathrm{m}$ SEDs were carefully analyzed and compared with a library of simulated-CVF spectra. The latter were created with a two-component model described hereafter. The role of the first component is to take into account the presence of the UIB features, usually exhibiting a canonical SED as it has been observed in various starforming galaxies (Dale et al. 2001; Roussel et al. 2001), so we used the average 5-16 $\mu \mathrm{m}$ SED of the M 51 galactic disk as a template spectrum. The second component, which reproduces the warm continuum observed in the long wavelength part of the CVF, was modeled using a black body with a temperature typically ranging from 150 to $300 \mathrm{~K}$. We also considered the use of a power law with a spectral index varying from $\alpha=1$ to $\alpha=2$, as well as black bodies modified by an emissivity law (see also Sturm et al. 2000; Förster Schreiber et al. 2003). However, given the similar shape of the power law and black body functions at these wavelengths and temperature regimes as well as the limited wavelength coverage of our spectra, we found that we were unable to discriminate between one of the two particular spectral shapes. As a result, we will restrict our analysis using simple black body fits. Selective reddening was then applied independently towards these two components (similarly to the approach followed by Tran et al. 2001), and a variety of extinction laws (Mathis 1990; Dudley \& Wynn-Williams 1997; Lutz 1999; Li \& Draine 2001) were explored assuming either a uniformed dust screen geometry $\left(F_{\lambda, \text { out }}=F_{\lambda, \text { in }} \times \exp ^{-\tau_{\lambda}}, \tau_{\lambda}=A_{\lambda} / 1.086\right)$ or a homogeneous mixture of dust grains and sources $\left(F_{\lambda, \text { out }}=F_{\lambda, \text { in }} \times\left(1-\exp ^{-\tau_{\lambda}}\right) / \tau_{\lambda}\right.$, $\tau_{\lambda}=A_{\lambda}$ /1.086). Varying the fraction of the UIB and continuum emission to the total mid-IR flux, a collection of CVF spectra was subsequently created and $\chi^{2}$-tested against our data to select the best simulated SED available in our library. Note that the strong ionic emission lines usually observed in starburst environments (Sturm et al. 2000) such as [NeII] at $12.8 \mu \mathrm{m}$ and [NeIII] at $15.6 \mu \mathrm{m}$ were not taken into account in our simulated CVF spectra as they are not included in our model. Moreover, at the spectral resolution of our CVF, [NeII] is blended with the $12.7 \mu \mathrm{m}$ UIB feature. Therefore the $12-13.2 \mu \mathrm{m}$ and $15-16 \mu \mathrm{m}$ wavelength ranges were omitted in our $\chi^{2}$ calculations.

In Fig. 4, we present the best fits we obtained for the four ISOCAM spectra, along with the decomposition into their two components. While the specific results which were derived for each source will be more thoroughly discussed in the next section, we highlight hereafter some general points of our findings, as well as a number of limitations related to our fitting procedures. 

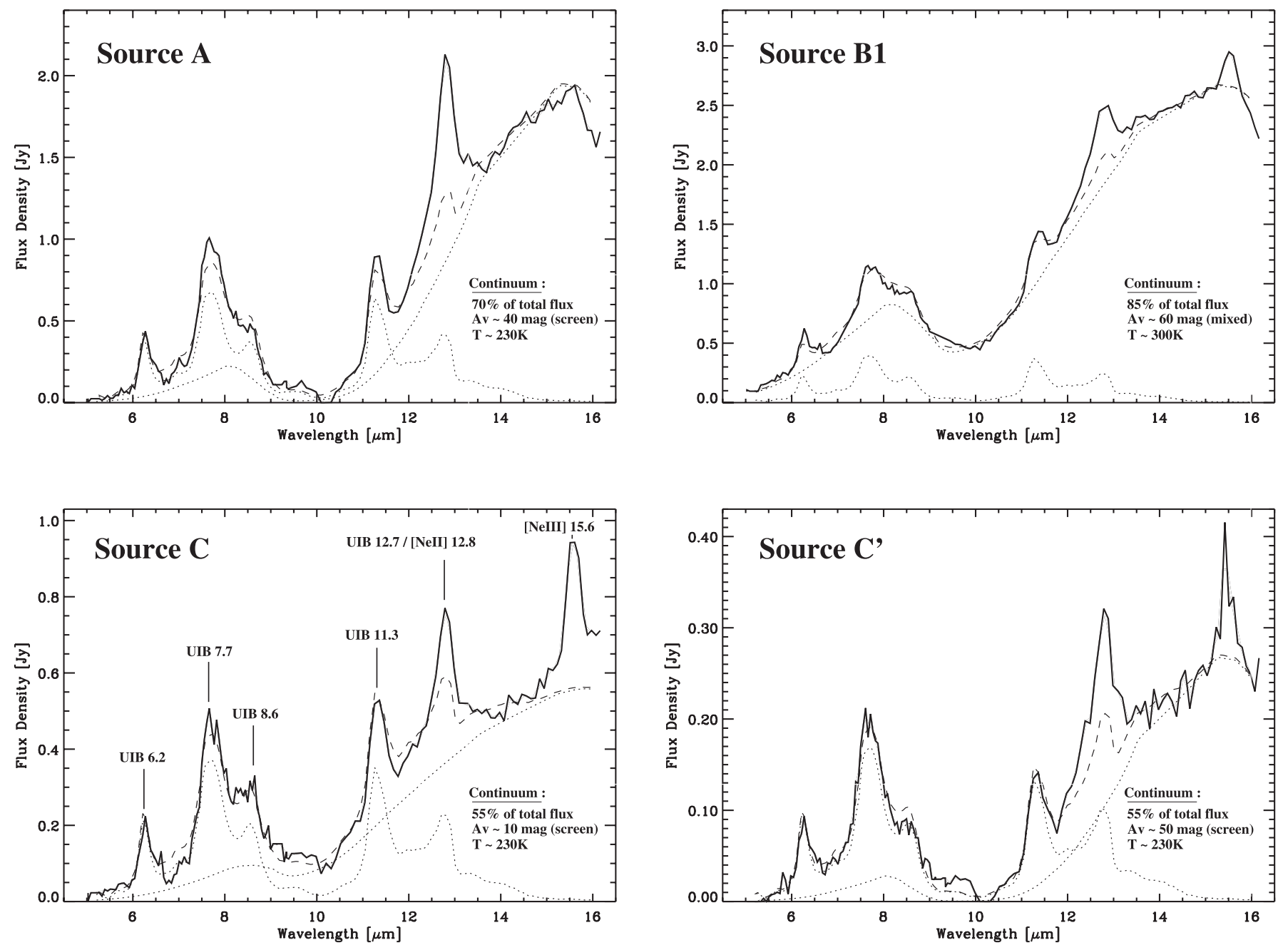

Fig. 4. Rest-frame mid-infrared spectra of the four main sources (A, B1, C and C') in Arp 299 (solid lines). They were obtained with a 4.5" $\times 4.5^{\prime \prime}$ aperture centered on each component, and they were corrected via a scaling factor to account for the extended flux of the PSF lying outside of the aperture. For each component, we have also plotted our best fit (dashed line) obtained with the combination of a UIB template and a black body continuum (dotted lines) extinguished with the law of Dudley \& Wynn-Williams (1997). On each panel, we indicate the physical properties of the continuum: its contribution to the mid-IR flux, the absorption magnitudes followed by the dust obscuration geometry (screen against mixed model), and the temperature of the fitting black body. We also found that the extinction level of the UIB component is negligible $\left(A_{\mathrm{V}} \lesssim 5 \mathrm{mag}\right)$ in the four sources. Note that the [NeII] $12.8 \mu \mathrm{m}$ and [NeIII]15.6 $\mu \mathrm{m}$ emission lines were not taken into account in our models.

One of our most striking results is that our best fits indicate an elevated extinction for the underlying continuum emission of sources $\mathrm{A}, \mathrm{B} 1$ and $\mathrm{C}^{\prime}$ and a very low extinction $\left(A_{\mathrm{V}} \lesssim 5 \mathrm{mag}\right)$ of the template which contributes to the UIB feature emission. This may suggest not only a physically different origin but also a different spatial distribution for the two components observed in our spectra. Such a picture could actually be understood if we consider that the continuum emission originates from deeply dust-enshrouded regions while the UIBs are more diffuse and have a widespread and surface distribution. Since the UIBs mostly trace areas of photo-dissociation surrounding HII regions, this would not be so surprising given the filamentary optical/near-IR emission (Malkan et al. 1998; Alonso-Herrero et al. 2000) and the extended gas streamers (Sargent \& Scoville 1991; Casoli et al. 1999) which have been observed in the in-between regions of Arp 299.
A potential limitation in our approach was revealed by the degeneracy originating from the unknown geometry of the dust in the obscured regions. For all sources but B1, we found that both the screen and the mixed models can actually lead to similarly good overall fits to the observed spectrum in terms of the $\chi^{2}$ minimization, even though the underlying assumptions for the geometry of the sources are different. For example, the continuum emission of source A can easily be reproduced either with a $230 \mathrm{~K}$ black body extinguished with $A_{\mathrm{V}}=40 \mathrm{mag}$ in a screen geometry (see Fig. 4), or with a much cooler component at $T=160 \mathrm{~K}$ with $A_{\mathrm{V}}=140 \mathrm{mag}$ assuming the mixed model. However, we wish to stress that the physical meaning of such single-temperature fits is rather uncertain since $i$ ) the observed spectrum is the result of no single grain population but a linear superposition of grains with a specific size distribution (see Dale et al. 2001), ii) the dust grains responsible for this warm continuum are not in thermal equilibrium, and 
Table 2. Intensities and ratio of the [NeII] $12.8 \mu \mathrm{m}$ and [NeIII] $15.6 \mu \mathrm{m}$ for Arp 299. The values of [NeII] were measured by Dudley (1999) and should be considered as upper limits as they may be contaminated by the $12.7 \mu \mathrm{m}$ PAH feature (see text).

\begin{tabular}{lccc}
\hline \hline Source & $\begin{array}{c}I([\mathrm{NeII}]) \\
\left(10^{-15} \mathrm{~W} \mathrm{~m}^{-2}\right)\end{array}$ & $\begin{array}{c}I([\mathrm{NeIII}]) \\
\left(10^{-15} \mathrm{~W} \mathrm{~m}^{-2}\right)\end{array}$ & $\frac{I([\mathrm{NeIII}])}{I([\mathrm{NeII}])}$ \\
\hline $\mathrm{A}$ & $<2$ & - & - \\
$\mathrm{B} 1$ & 0.7 & $>1.3$ & $\geq 1.8$ \\
$\mathrm{C}$ & 2.0 & 1.3 & $\geq 0.65$ \\
$\mathrm{C}^{\prime}$ & $<0.9$ & 0.5 & $>0.55$ \\
\hline
\end{tabular}

iii) the presence of temperature gradients in the close vicinity of the mid-IR sources is quite likely. Therefore, a more meaningful approach would rather be to compare the four fitted spectra to each other after having primarily defined a given geometry for the dust obscuration (see Sect. 5 for such comparisons). Furthermore, we note that both the fraction of UIBs to the total mid-IR flux and their negligible extinction measured using our fitting procedure are roughly independent of the screen or mixed model assumption.

To investigate the hardness of the radiation field in the different regions of the system we can use the ratio of the ionic Neon lines [NeII]12.8 $\mu \mathrm{m}$ and [NeIII] $15.6 \mu \mathrm{m}$. Even though we can easily measure the [NeIII] line, the spectral resolution of the CVF $(R \sim 40)$ is just too low to distinguish the [NeII] $12.8 \mu \mathrm{m}$ line from the $12.7 \mu \mathrm{m}$ PAH band. As a result we decided to use the [NeII] values of the system measured by Dudley (1999) who calculated the intensity of the [NeII] lines in all sources of Arp 299. His $R \sim 60$ spectrograph can almost resolve the [NeII] line, and the 5.5 " circular aperture he used is only $\sim 15 \%$ larger than the aperture used to extract our ISOCAM/CVF spectra presented in Fig. 4. Our results are presented in Table 2. In principle, the values of [NeII] may still contain some contribution from the $12.7 \mu \mathrm{m}$ PAH feature, thus making the [NeIII]/[NeII] ratio a lower limit. However, this contribution must be small since the sum of line intensities measured by Dudley (1999) in the regions $\mathrm{B} 1, \mathrm{C}$, and $\mathrm{C}^{\prime}$ is consistent with the high spectral resolution ISO/SWS measurements of Thornley et al. (2000). We note an inconsistency between the sum of our measurements on $\mathrm{B} 1, \mathrm{C}$ and $\mathrm{C}^{\prime}$ and the integrated measurement presented in Thornley et al. (2000). Whether their value underestimated or our overestimated is not easy to determine, and spectroscopic observations of this target with SIRTF will resolve this issue.

One of the great advantages of the ISOCAM/CVF observations is that we can extract narrower-band images $(\Delta \lambda \sim 1 \mu \mathrm{m})$ of our target, effectively constructing two dimensional maps of specific spectral features. Several maps of selected spectral regions were created for Arp 299 and are displayed in Fig. 5. The first is the hot dust continuum at $5 \mu \mathrm{m}$, which could be due to either thermal emission from an AGN or the Rayleigh-Jeans tail of the photospheric emission from an old stellar population, or fluctuating small dust grains without aromatic features (Helou et al. 2000). The integrated emission of the $6.2 \mu \mathrm{m}$ UIB is also presented, since it isolates the emission from the photo-dissociation regions of the merger and despite the fact that it is weaker than the $7.7 \mu \mathrm{m}$ UIB it is much less affected by the depth of the $9.7 \mu \mathrm{m}$ silicate band (see discussion in Laurent et al. 2000). We also mapped the total flux within the band of the silicate absorption at $9.7 \mu \mathrm{m}$, and that of the ionic [NeIII] $15.6 \mu \mathrm{m}$ emission line which is indicative of the hardness of the radiation field (e.g., Thornley et al. 2000). For the latter and the $6.2 \mu \mathrm{m}$ UIB, we identified the corresponding spectral feature within each pixel of the CVF images, and carefully removed the underlying continuum so as to only keep the contribution of the given emission line (see Laurent et al. 2000 and Le Floc'h et al. 2001 for an illustration of the method). The dominant sources of mid-IR emission (A, B and C) are clearly apparent in all maps, even though their relative strength varies at each wavelength. However, the image of the ionic [NeIII] $15.6 \mu \mathrm{m}$ line remains indicative of the spatial distribution of this feature. The spectra of individual pixels are noisier than any integrated spectra and the determination of the continuum pixel-per-pixel can be altered by this noise. Moreover, especially in the case of source B1, the depression in the SED over $16 \mu \mathrm{m}$ can lead to a bad estimation of the continuum and then to a bad determination of the flux in the line. So, integrated spectra on each main source are more reliable in term of relative flux. Even if $\mathrm{C}$ appears as the dominant source in the [NeIII] $15.6 \mu \mathrm{m}$ image of Fig. $5 \mathrm{~d}$, B1 remains the brighest source in this line.

\section{Discussion}

\subsection{The nuclear region of IC 694}

In our mid-IR images, IC 694 (source A) appears as a bright source corresponding to the radio peak observed at 6 and $20 \mathrm{~cm}$ by Gehrz et al. (1983). As pointed out (see Alonso-Herrero et al. 2000; Charmandaris et al. 2002 and references therein), unlike the infrared view, images in the UV and visible do not reveal any clear optical counterpart at this position, while large quantities of high density molecular gas are concentrated within the central 500 pc (Sargent \& Scoville 1991; Casoli et al. 1999). This gas fuels a massive starburst as evidenced by a supernova rate of $0.65 \mathrm{yr}^{-1}$, nearly five times the combined rate observed in B and C (Alonso-Herrero et al. 2000). This large gas reservoir can easily explain the high extinction toward the nucleus. In the optical, a dust lane runs in the southeast/northwest direction and "patchy" emission from surface star forming regions is seen in an extended area around the nucleus (Malkan et al. 1998). Not surprisingly, those same regions contribute to the $6.2 \mu \mathrm{m}$ UIB emission and, as we observe in Fig. 5b, the angular size of source A is considerably larger at this wavelength (see also Fig. 2 which shows this diffuse emission).

Our mid-IR spectrum of this source (see Fig. 4) reveals a strong continuum with a slope past $10 \mu \mathrm{m}$ steeper than in any other component of the system. Moreover, the $9.7 \mu \mathrm{m}$ silicate band appears saturated. The continuum emission from A can be fitted with a $230 \mathrm{~K}$ black body absorbed by $A_{\mathrm{V}} \sim 40$ mag assuming a uniform screen of dust. We also note that the longer wavelength part of the spectrum seems to decrease again past $15.5 \mu \mathrm{m}$. The same behavior can be seen in source B. Since we do know from multiple observations between 20 and $38 \mu \mathrm{m}$ 


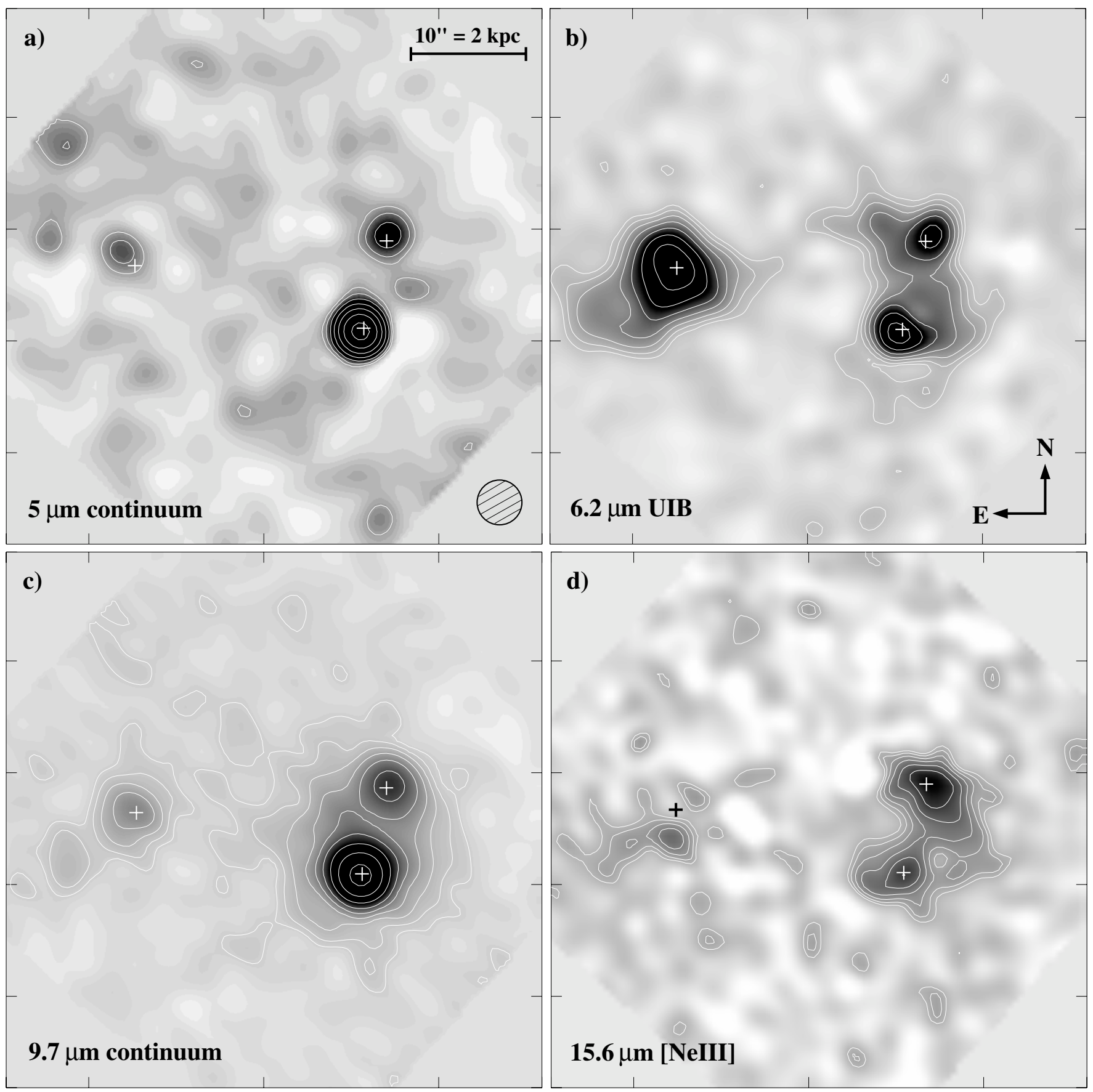

Fig. 5. Inverted grey-scale images of Arp 299 in selected spectral regions, depicting a) the hot continuum emission observed at $5 \mu \mathrm{m}$, b) the photo-dissociation regions obtained by integrating the mid-IR emission within the $6.2 \mu \mathrm{m} \mathrm{UIB,} \mathrm{c)} \mathrm{the} \mathrm{continuum} \mathrm{emission} \mathrm{integrated} \mathrm{over} \mathrm{a}$ $\Delta \lambda \sim 1 \mu \mathrm{m}$ bandwidth in the range of the silicate absorption at $9.7 \mu \mathrm{m}$, and $\mathbf{d}$ ) the distribution of the ionic [NeIII] emission line at $15.6 \mu \mathrm{m}$, after subtraction of the underlying continuum. This last image must be considered carefully because the continuum per pixel on source B may have been badly estimated due to the low signal to noise ratio of the emission in the line and the probable influence of the wide $18 \mu \mathrm{m}$ silicate absorption band. For each image, the overlaid contours have been set with logarithmic spacing. By increasing order of wavelengths, the minimum levels are respectively $0.44,0.18,0.22$ and $0.49 \mathrm{mJy} \mathrm{arcsec}^{-2}$ while the maxima are $10.7,2.0,15.5$ and $1.5 \mathrm{mJy}$ arcsec ${ }^{-2}$. The positions of the brightest sources (A, B1 and C) over the 5-16 $\mu \mathrm{m}$ wavelength range are marked with crosses. The four images have the same orientation. The average FWHM of the ISOCAM PSF over the full CVF spectral range $\left(\sim 4^{\prime \prime}\right)$ is indicated in panel a) as well as the scale in arcsec and kpc.

that the spectrum of A rises quickly making it the dominant source of the IR luminosity of the system (Charmandaris et al. 2002), it is reasonable to attribute this brief decrease in flux to the presence of the $18 \mu \mathrm{m}$ silicate feature. Indeed, as shown by Dudley \& Wynn-Williams (1997), the two silicate bands are proportional in strength/depth so, given the observed shape of the $9.7 \mu \mathrm{m}$ feature, the $18 \mu \mathrm{m}$ one would have to be strong too. Unfortunately given the limited wavelength coverage of the 
CVF and the fact that memory effects of the detector are more pronounced at the longer wavelengths which are observed at the beginning of the scan, we can not elaborate more on its actual strength. We will have to wait for the Infrared Spectrograph (IRS) aboard the Space Infrared Telescope Facility (SIRTF) which will provide complete wavelength coverage up to $40 \mu \mathrm{m}$ to address this issue more accurately.

A unique aspect of IC 694 that separates it from all other areas in the galaxy is the weak [NeIII] $15.6 \mu \mathrm{m}$ emission. As we can see from Fig. 4a, even though a suspicious feature at $15.6 \mu \mathrm{m}$ could be the [NeIII] line, its equivalent width is very small and makes it practically impossible to measure with our resolution. This can not be easily explained by advocating that the blue wing of the $18 \mu \mathrm{m}$ silicate feature extends to the location of [NeIII] reducing the line flux. If simple extinction was the culprit then how could one detect the line in both regions B1 and $\mathrm{C}^{\prime}$ where even higher values of $A_{\mathrm{V}}$ are found? Furthermore, why does it seem that the spatial distribution of the faint [NeIII] avoids the nucleus but is associated with the dusty filaments to the southeast of source A?

The fact that the mid-IR neon lines of IC 694 have also been observed with ISO/SWS with superior spectral resolution by Thornley et al. (2000) can help us explore these questions. Using a considerably larger aperture $\left(14^{\prime \prime} \times 27^{\prime \prime}\right)$ these authors easily detected both [NeII]12.8 $\mu \mathrm{m}$ and [NeIII] $15.6 \mu \mathrm{m}$ toward IC 694. They measured a [NeIII]/[NeII] ratio of 0.29 , a factor $\sim 2.5$ times lower than what was found for the whole of NGC 3690 (combined sources B and C). Modeling by Thornley et al. (2000) has shown that low values of the $[\mathrm{NeIII}] /[\mathrm{NeII}]$ ratio can be explained in terms of a larger age of the starburst, lower limit in the upper mass function cut off, higher metallicity, or a reduced ionization parameter $U$. Our observations are actually consistent with all these possibilities if we consider that IC 694 contains the larger fraction of molecular gas and cold dust and dominates the IR luminosity of Arp 299. This implies that the starburst in source A has been taking place for a longer period of time than in sources $\mathrm{B}$ and $\mathrm{C}$ since the former seems to be closer to dynamical equilibrium than the two sources in NGC 3690 which are even more disturbed and obviously still strongly interacting (Casoli et al. 1999). One could further speculate that the geometry in A is different from the single cluster case of Thornley et al. (2000). The spatial extent associated with the age of the starburst indicates that the distribution of the enshrouded stars is more random forming clusters resembling the mix gas/star cluster case. Furthermore, more generations of stars must have been created in IC 694, and passing through their AGB phase, they produced the dust we observe and also lead to a higher metallicity in the region. As we will discuss in the following section this is not the case for $\mathrm{C}^{\prime}$ which must harbor the youngest $\mathrm{OB}$ stars in the system (Soifer et al. 2001).

If the above scenario is correct then the reason why we detect $[\mathrm{NeIII}]$ in the southeast outskirts of source A, and not in the center, is simply due to the fact that these are the regions where young stars form along the tidal filaments (Malkan et al. 1998). In these filaments, the geometry is much more favorable so that "bubbles" in the interstellar medium form easier exposing the high ionization radiation of some underlying recently formed massive stars.

\subsection{On the mid-IR properties of sources $C$ and $C^{\prime}$}

The shape of the mid-IR SED of these regions (see Fig. 4) is consistent with those of star-forming regions in other extragalactic sources, where a hot thermal continuum strongly affects the PAH emission. Furthermore, the values of the midIR diagnostic flux ratios LW3/LW2 (see Table 1) are similar to those found in regions dominated by young massive stars (see Laurent et al. 2000). As a result, our data provide further support to the conclusions of other authors (Soifer et al. 2001 and references therein) that $\mathrm{C}$ and $\mathrm{C}^{\prime}$ host extraordinary extra-nuclear starbursts.

Although it is probable that some emission from $\mathrm{C}$ could contribute to $\mathrm{C}^{\prime}$ because of their small angular separation, the fact that their measured absorptions are different indicates this is not a very likely event. Our modeling of the extinction suggests that the environments for both sources $\mathrm{C}$ and $\mathrm{C}^{\prime}$ are similar, with a higher extinction toward $C^{\prime}$. For both, as for source $\mathrm{A}$, the observed spectrum can be reproduced by the simple superposition of a UIB contribution and a black body heated to $\sim 230 \mathrm{~K}$ in order to account for the hot dust continuum which dominates above $10 \mu \mathrm{m}$. The estimated extinction for sources $\mathrm{C}$ and $\mathrm{C}^{\prime}$ is $A_{\mathrm{V}}$ of 10 and 50 mag respectively assuming a foreground screen geometry.

The hardness of the UV radiation field in these sources can be characterized by the $[\mathrm{NeIII}] /[\mathrm{NeII}]$ ratio. Source $\mathrm{C}$ presents a ratio of $\geq 0.65$ and $C^{\prime}$ a ratio $\geq 0.55^{3}$, both greater than the one measured in component A (0.29 from Thornley et al. 2000). This would indicate that the UV radiation field, and consequently the fraction of young massive stars is higher in $\mathrm{C}$ and $\mathrm{C}^{\prime}$ than in A, leading to younger starbursts in these regions, in agreement with the conclusions of Soifer et al. (2001) as well as of Alonso-Herrero et al. (2000) who derived $\sim 5 \mathrm{Myr}, 4 \mathrm{Myr}$ and $\sim 11$ Myr for $\mathrm{C}, \mathrm{C}^{\prime}$ and A respectively (based on an evolutionary starburst model using parameters derived from their observations).

\subsection{The nature of source $B$ : Is there mid-IR evidence for an AGN?}

As we mentioned earlier, B1 appears to be the brightest source of the system in the 5 to $16 \mu \mathrm{m}$ spectral range and remains unresolved by our observations. While our spatial resolution is not sufficient to unambiguously resolve B1 and B2, the fact that the centroid of the source located at B position coincides very well with the $6 \mathrm{~cm}$ radio source observed by Gehrz et al. (1983) indicates reasonably that we actually map mainly the emission coming from B1. This is in agreement with higher spatial resolution ground-based observations from Soifer et al. (2001)

\footnotetext{
3 We must be careful considering [NeIII] in source $\mathrm{C}^{\prime}$. We notice that the emission in this line is only marginally mapped at the $\mathrm{C}^{\prime}$ position (see Fig. 5). Because $C$ and $C^{\prime}$ are separated only by $5^{\prime \prime}$, the feature we observe in the $\mathrm{C}^{\prime}$ spectrum may be a relic of the extended wings of the PSF $\left(F W H M \sim 4.5^{\prime \prime}\right)$ centered on source $\mathrm{C}$.
} 
who resolved B1 and B2 but showed that, despite the fact that B2 dominates the flux below $2 \mu \mathrm{m}$ (see also Wynn-Williams et al. 1991; Gallais 1991), its emission decreases with increasing wavelength and it is actually $\sim 35$ times fainter than B1 at $12.5 \mu \mathrm{m}$. Consequently, all our conclusions on the mid-IR characteristics of source $\mathrm{B}=\mathrm{B} 1+\mathrm{B} 2$ would actually reflect the properties of B1.

Until recently, most researchers had focussed on the starburst properties of this region, given the absence of any evidence for an active nucleus. Based on the $\mathrm{H} \alpha$ (see Fig. 3 of Alonso-Herrero et al. 2000) and CO observations (Casoli et al. 1999; Aalto et al. 1997), it is clear that B1 contains a large amount of ionized gas, as well as cold molecular gas which could sustain massive star formation activity for more than $10^{8}$ years. Our observations though indicate that the physical characteristics of $\mathrm{B} 1$, as displayed by its spectrum, make it stand out in the mid-IR in two ways.

First, B1 displays the strongest continuum emission over the whole 5-16 $\mu \mathrm{m}$ range with only a weak contribution due to PAH emission (see Fig. 4). Using our model, we find that this continuum can be best fitted with a $300 \mathrm{~K}$ black body extinguished by $A_{\mathrm{V}} \sim 60 \mathrm{mag}$ in a mixed-model geometry and accounts for $85 \%$ of the total mid-IR emission of B1. Despite the high extinction derived from our model, B1 remains the brightest source of the system in the $9.7 \mu \mathrm{m}$ silicate absorption band and its "hot continuum" at $\sim 5 \mathrm{~m}$ is $118 \mathrm{mJy}$, almost 5 times higher than source A which dominates the global IR luminosity of the whole system.

Second, the $[\mathrm{NeIII}] /[\mathrm{NeII}]$ ratio of this source is at least 1.8 (see Table 2), a value considerably greater than the ratios measured in the Thornley et al. (2000) starburst sample which typically ranges from 0.05 to 1 , with the only exception of the low metallicity systems NGC 5253 and II Zw 40 (with values of 3 and 12 respectively). Clearly, the radiative environment in B1 deviates from the one seen in classical starburst galaxies, and it appears to resemble more what is observed in young galactic HII regions such as W 51 (Thornley et al. 2000). Sturm et al. (2002) reports measurements in the Neon lines for Seyfert galaxies which clearly indicate a higher ratio in pure Seyfert galaxies (usually $>1$ with a maximum of 2.8 in their sample) than in mixed Seyfert/starburst systems $(<0.6$ typically). On the other hand, the ratio $[\mathrm{NeIII}] /[\mathrm{OIV}]$ is usually low $(<1.5)$ in Seyfert galaxies as computed from values reported by Sturm et al. (2002) while it is higher in starburst galaxies, ranging from 5 to $>30$ (see Verma et al. 2003). Assuming that $\mathrm{C}$ and $\mathrm{C}^{\prime}$ contributes to the flux measured in [OIV] by Verma et al. (2003), B1 then presents a ratio [NeIII]/[OIV] $>13$. Even if those ratios are not criteria used to discriminate Seyfert and starburst galaxies, the association of a high [NeIII]/[NeII] with a high $[\mathrm{NeIII}] /[\mathrm{OIV}]$ in source B1 makes it of very intriguing origin.

The fact that no UV emission is detected from B1 is further evidence of the high obscuration in the region. In this context, the PAH features could be attributed to individual heating sources distributed around B1, in a diffuse component on the line of sight, in such a way that the filling factor of their corresponding Stromgren spheres is high and hence there is a smaller volume available for the photo-dissociation regions to contribute to the PAH flux (see Alonso-Herrero et al. 2000, Fig. 7b).

However, hard UV photons can also be produced by an AGN. Based on a diagnostic diagram which compares the ratio of the continuum at $14-15 \mu \mathrm{m}$ to the continuum at $5.1-6.8 \mu \mathrm{m}$ versus the ratio of the strength of the $6.2 \mu \mathrm{m}$ feature to the continuum at 5.1-6.8 $\mu \mathrm{m}$, Laurent et al. (2000) attempted to quantify the AGN/starburst contribution in the mid-IR. For source B1, their Fig. 6 suggests that almost $40 \%$ of the mid-IR emission could originate from a very embedded AGN. The telltale sign would be the emission observed via the "hot continuum" at $5 \mu \mathrm{m}$ as illustrated in our Fig. 5. Even though the midIR evidence was not strong enough to propose the presence of an AGN detection, their suggestion was in fact proven true beyond any doubt with the recent Beppo-Sax X-ray observations of Arp 299 by Della Ceca et al. (2002). These authors explained the hard X-ray emission and spectral shape/luminosity of the system with a buried Compton-thick AGN of intrinsic luminosity $L_{0.5-100 \mathrm{keV}} \simeq 1.9 \times 10^{43} \mathrm{erg} \mathrm{s}^{-1}$, completely absorbed in the visible and in the IR $\left(A_{\mathrm{V}}>1000 \mathrm{mag}\right)$. Their result, as well as further work based on Chandra data which places the AGN in B1 (Zezas et al. 2003), readily explains our inability to probe the center of B1 in the optical and the UV.

An interesting point which remains to be explored in the future is whether the AGN actually defines the location of a galactic nucleus in NGC 3690. The reason for this question is that B2 is the most extended source in the visible as seen in the HST WFPC2 $(0.59 \mu \mathrm{m})$ images and the brightest one in the near-infrared, while B1 remains unresolved or marginally resolved (Alonso-Herrero et al. 2000). Moreover, unlike B1, B2 does display a high $\mathrm{CO}$ index characteristic of a population of late-type stars (Satyapal et al. 1999). Based on its near-IR colors and its size at these wavelengths, B2 appears to be mainly composed of a mix of old stars, gas and dust. If this source is a relic of an older single isolated starburst region outside the nucleus traced by B1, it should have been spatially extended and of an extraordinary strength.

If, on the other hand, the nucleus of the galaxy is in B2, as suggested by the near-IR colors, then this would imply that B1 harbors not only massive star forming regions but also a "runaway" super-massive black hole (SMBH). Did this SMBH originate from a companion which was accreted in the system at an earlier stage and it is now accreting gas becoming visible as an AGN? We do observe a high concentration of gas outside - but close to - the B1 and B2 regions of NGC 3690 and their kinematics imply that they are tidally connected with streamers originating from IC 694 (Casoli et al. 1999). Alonso-Herrero et al. (2000) though derived a dynamical mass for B1 $(M>$ $\left.4 \times 10^{8} M_{\odot}\right)$ similar to the one found in $\mathrm{B} 2\left(M \sim 6 \times 10^{8} M_{\odot}\right.$, from Shier et al. 1996) which would be hard to explain if both of them had always been part of a single galaxy. Our mid-IR observations do not allow us to discriminate between the two interpretations. Only high spatial resolution information on the kinematics of those components will help us shed some light to these issues.

In any case, NGC 3690 has been the host of extraordinary star-forming events and exhibits a distinct mid-infrared spectral energy distribution unlike any other in other extragalactic 
objects, even unambiguous AGNs such as NGC 1068 (Le Floc'h et al. 2001) or Centaurus A (Mirabel et al. 1999).

\section{Conclusions}

Our mid-IR spectroscopy of Arp 299 over the range 5 to $16 \mu \mathrm{m}$ has revealed new information on the properties of this intriguing interacting system. Based on our analysis, we conclude that:

1. Large quantities of dust are present in the system as indicated by the hot continuum observed above $10 \mu \mathrm{m}$ in all sources and by the high extinction derived by our model. We find that, assuming a uniform foreground screen model, we can reproduce the mid-IR SED and the $9.7 \mu \mathrm{m}$ silicate absorption band with an $A_{\mathrm{V}}$ of $\sim 40$ mag in the central region of IC 694 (source A), 10 mag for source C, and $\sim 50$ mag for $\mathrm{C}^{\prime}$, the dominant sources in the galactic disks overlap region. A best fit with an $A_{\mathrm{V}}$ of $\sim 60 \mathrm{mag}$ is derived in the central region of NGC 3690 (source B1) using a homogeneous mixture of dust with radiating sources.

2. The nuclear region of IC 694 harbors enshrouded massive star forming activity. No evidence for an active galactic nucleus is found in our mid-IR spectra.

3. We confirm that $\mathrm{C}$ and $\mathrm{C}^{\prime}$ are sites of recent starburst events based on the elevated values of the [NeIII] $15.6 \mu \mathrm{m}$ flux and the high $[\mathrm{NeIII}] /[\mathrm{NeII}]$ ratio, which is similar to those of classical young starbursts.

4. Source B is characterized by a unique mid-IR spectrum which is dominated by a hot dust continuum extending shortward of $5 \mu \mathrm{m}$, and which is the strongest of the Arp 299 system. Given the ample presence of dust and the strength of the radiation field, this continuum is consistent with the recent Chandra/Beppo-SAX results which favor the presence of a Compton thick AGN at this location.

Acknowledgements. We thank the HST team and PIs of the proposals who provided the WFPC2 NICMOS and FOC images made with the NASA/ESA Hubble Space Telescope and obtained from data archives at the Space Telescope Science Institute. STScI is operated by the Association of Universities for Research in Astronomy, Inc. under the NASA contract NAS 5-26555. VC would like to thank F. Casoli (Obs. de Paris) for useful discussions on the CO kinematics, A. Zezas (CfA/Harvard) for information on the Chandra results prior to publication and acknowledges the financial support of JPL contract 960803.

\section{References}

Aalto, S., Radford, S. J. E., Scoville, N. Z., \& Sargent, A. I. 1997, ApJ, 475, L107

Abergel, A., Bernard, J.-P., Boulanger, F., et al. 1996, A\&A, 315, L329

Athey, A., Bregman, J., Bregman, J., et al. 2002, ApJ, 571, 272

Augarde, R., \& Lequeux, J. 1985, A\&A, 147, 273

Alonso-Herrero, A., Rieke, G. H, Rieke, M. J., \& Scoville, N. Z. 2000, ApJ, 532, 845

Casoli, F., Willaime, M.-C., Viallefond, F., \& Gerin, M. 1999, A\&A, 346, 663

Cesarsky, C. J., Abergel, A., Agnèse, P., et al. 1996, A\&A, 315, L32
Charmandaris, V., Laurent, O., Mirabel, I. F., et al. 1999, A\&A, 341, 69

Charmandaris, V., Stacey, G. J, \& Gull, G. 2002, ApJ, 571, 282

Dale, D. A., Helou, G., Contursi, A., Silbermann, N. A., \& Kolhatkar, S. 2001, ApJ, 549, 215

Della Ceca, R., Ballo, L., Tavecchio, F., et al. 2002, ApJ, 581, L9

Dudley, C. C., \& Wynn-Williams, C. G. 1993, ApJ, 407, L65

Dudley, C. C., \& Wynn-Williams, C. G. 1997, ApJ, 488, 720

Dudley, C. C. 1999 , MNRAS, 307, 553

Fabbiano, G., Kim, D.-W., \& Trinchieri, G. 1992, ApJS, 80, 531

Förster Schreiber, N. M., Sauvage, M., Charmandaris, V., et al. 2003, A\&A, 399, 833

Gallais, P. 1991, Thèse de Doctorat de l'Université Paris VII

Gallais, P., Laurent, O., Charmandaris, V., et al. 1999, in The Universe as seen by ISO, ESA SP-427, 881

Gehrz, R. D., Sramek R. A., \& Weedman, D. W. 1983, ApJ, 267, 551

Helou, G., Nanyao, Y. L., Werner, M. W., Malhotra, S., \& Silberman, N. 2000, ApJ, 532, L21

Hibbard, J. E., \& Yun, M. S. 1999, AJ, 118, 162

Lai, O., Rouan, D., Rigaut, F., Doyon, R., \& Lacombe, F. 1999, A\&A, 351,834

Laurent, O., Mirabel, I. F., Charmandaris, V., et al. 2000, A\&A, 359, 887

Le Floc'h, E., Mirabel, I. F., Laurent, O., et al. 2001, A\&A, 367, 487

Li, A., \& Draine, B. T. 2001, ApJ, 554, 778

Lutz, D. 1999, in The Universe as seen by ISO, ESA SP-427, 623

Malkan, M. A., Gorjian, V., \& Tam, R. 1998, ApJS, 117, 25

Mathis, J. S. 1990, ARA\&A, 28, 37

Meurer, G. R., Heckman, T. M., Leitherer, C., et al. 1995, AJ, 110, 2665

Mihos, J. C., \& Hernquist, L. 1996, ApJ, 464, 641

Mirabel, I. F., Vigroux, L., Charmandaris, V., et al. 1998, A\&A, 333, L1

Mirabel, I. F., Laurent, O., Sanders, D., et al. 1999, A\&A, 341, 667

Rouan, D., Clénet, Y., \& Lai, O. 1999, in Astronomy with adaptive optics: present results and future programs, ESO Conf. and Workshop Proc., 56, 411

Roussel, H., Sauvage, M., Vigroux, L., \& Bosma, A. 2001, A\&A, 372, 427

Sanders, D. B., \& Mirabel, I. F. 1996, ARA\&A, 34, 749

Sargent, A., \& Scoville, N. 1991, ApJ, 366, L1

Satyapal, S., Watson, D. M., Forrest, W. J., et al. 1999, ApJ, 516, 704

Soifer, B. T., Neugebauer, G., Matthews, K., et al. 2001, AJ, 122, 1213

Shier, L. M., Rieke, M. J., \& Rieke, G. H. 1996, ApJ, 470, 222

Starck, J.-L., Abergel, A., Aussel, H., et al. 1999, A\&AS, 134, 135

Sturm, E., Lutz, D., Tran, D., et al. 2000, A\&A, 358, 481

Sturm, E., Lutz, D., Verma, A., et al. 2002, A\&A, 393, 821

Thornley, M. D., Förster-Shreiber, N. M., Lutz, D., et al. 2000, ApJ, 539, 641

Tran, Q. D., Lutz, D, Genzel, R., et al. 2001, ApJ, 552, 527

Verma, A., Lutz, D., Sturm, E., et al. 2003, A\&A, 403, 829

Vigroux, L., Mirabel, I. F., Altiéri, B., et al. 1996, A\&A, 315, L93

Weedman, D. W. 1972, ApJ, 171, 5

Windhorst, R. A., Taylor, V. A., Jansen, R. A., et al. 2002, ApJS, 143, 113

Wynn-Williams, C. G., Eales, S. A., Beklin, E. E., et al. 1991, ApJ, 377,426

Zezas, A. L., Georgantopoulos, I., \& Ward, M. J. 1998, MNRAS, 301, 915

Zezas, A. L., Ward, M. J., \& Murray, S. S. 2003, ApJ, 594, L31 